\title{
IoT based heart monitoring and alerting system with cloud computing and managing the traffic for an ambulance in India
}

\author{
Khushboo Bhagchandani ${ }^{1}$, D. Peter Augustine ${ }^{2}$ \\ ${ }^{1}$ Department of Computer Science, CHRIST (Deemed to be University), India \\ ${ }^{2}$ Faculty of Computer Science, CHRIST (Deemed to be University), India
}

\section{Article Info \\ Article history: \\ Received Dec 31, 2018 \\ Revised Jun 29, 2019 \\ Accepted Jul 3, 2019}

\section{Keywords:}

Arduino

Healthcare

Heart attack

IOT

\begin{abstract}
Global Burden of Disease Report, released in Sept 2017, shows that Cardiovascular Diseases caused 1.7 million deaths (17.8\%) in 2016 and it is the leading cause of deaths in India [1]. According to the Indian Heart Association, 25\% of all heart attacks happen under the age of 40 . In most cases, the initial heart attacks are often ignored. Even post-diagnosis, as per government data [2], 50\% of heart attack cases reach the hospital in more than 400 minutes against the ideal window time of 180 minutes; post which damage is irreversible. The delay is often attributed to delay in reaching a hospital or receiving primary aid. In India, traffic conditions also add to the grimace of the situation. Although the government is taking various measures; a holistic solution is required to minimize the delay at each of the steps like accessing the patient situation, contacting the Medical aid or making available the nearest aid possible. In this paper, we aim at providing the holistic solution using the Internet of Things technology (IOT) along with data analytics. IoT enables real-time capturing and computation of medical data from smart sensors built-in wearable devices. The amalgamation of Internet-based services with Medical Things (Smart sensors) enhance the chances of survival of patients. The proposed system analyses the inputs collected from the sensors fit with the patients prone to cardiovascular diseases to ascertain the emergency situation. In addition, to these data, the system also considers age, maximum and minimum heart rate. Based on computational results received from the input parameters, the system triggers the alert to emergency contacts such as the close relatives of the patient, doctors, the hospitals and nearby ambulance. The proposed system combines with the optimized navigation platform to guide the medical assistance to find the fastest route.
\end{abstract}

Copyright () 2019 Institute of Advanced Engineering and Science. All rights reserved.

\section{Corresponding Author:}

Khushboo Bhagchandani,

Department of Computer Science,

CHRIST (Deemed to be University), India.

Email: angelforallkhushi@gmail.com

\section{INTRODUCTION}

Due to change in lifestyle, Indians are more susceptible to Cardiovascular Diseases. These are also silent killer as CVDs are mostly associated with the older age. Although, the recent studies have proven that almost a quarter of heart attacks occur under the age of 40. This situation is further aggravated by the stress, sedentary lifestyle, and diseases like diabetes. Hence, it is imperative to monitor the health on a daily basis and take medical treatment for the same if required. The Figure 1 shows the different ways to measures the heart attack, there are multiple sensors in the market, and some as shown above. 


\begin{tabular}{|c|c|c|c|}
\hline Clinical applications & Location & Type of Sensor & Marker \\
\hline \multirow{10}{*}{$\begin{array}{l}\text { Cardiopulmonary \& } \\
\text { Vascular Monitoring }\end{array}$} & \multirow[t]{3}{*}{ Wrist } & Ultrasound & Blood pressure \\
\hline & & Multi-variable (AMON) & $\begin{array}{l}\text { Blood pressure, blood oxygen saturation, body } \\
\text { temp, heart rhythm }\end{array}$ \\
\hline & & $\begin{array}{l}\text { Photoplethysmographic and } \\
\text { electrocardiograph }\end{array}$ & Blood pressure and heart rtythm \\
\hline & \multirow[t]{2}{*}{ Finger (ring sensor) } & Optical (heart rate) & \multirow[t]{2}{*}{ Heart rate and temperature } \\
\hline & & $\begin{array}{l}\text { Radio-frequency identification } \\
\text { (pulse \& temp). }\end{array}$ & \\
\hline & Arm or thigh & $\begin{array}{l}\text { Microwave reflectomettic } \\
\text { cardiopulmonary }\end{array}$ & Heart rate variability as a method to evaluate stress \\
\hline & \multirow{2}{*}{$\begin{array}{l}\text { Can be used with various } \\
\text { equipment units }\end{array}$} & Optical absorption sensor & \multirow{2}{*}{$\begin{array}{l}\text { Blood oxygen, heart rate and rhythm, fatigue levels } \\
\text { and exercise stress }\end{array}$} \\
\hline & & ECG electrodes & \\
\hline & Phone adapter & Singlechannel ECG & Heart rate and rhythms \\
\hline & Seat belt of a car & Wire-type strain gauge & Heart rate and respiration rate \\
\hline \multirow{3}{*}{$\begin{array}{l}\text { Glucose Home } \\
\text { Monitoring }\end{array}$} & Arm & Multi-variable & Blood glucose \\
\hline & Subcutaneous & Glucose & Tissue glucose \\
\hline & Eye & Glucose & Ocular glucose \\
\hline \multirow[t]{4}{*}{$\begin{array}{l}\text { Neurological Function } \\
\text { Monitoring }\end{array}$} & Clothes & Inertial sensors and accelerometers & $\begin{array}{l}\text { Walking distance, stride distance, and step count } \\
\text { during stair ascent and descent }\end{array}$ \\
\hline & Visual feedback-glasses & Inertia & Walking speed and stride length \\
\hline & $\begin{array}{l}\text { Auditory feedback- } \\
\text { headphones }\end{array}$ & & \\
\hline & Wrist or ankle & Accelerometer and motion & Seizure activity \\
\hline $\begin{array}{l}\text { Physical Therapy and } \\
\text { Rehabilitation }\end{array}$ & Ankle & Pedometers and accelerometer & Walking distance and step counts \\
\hline
\end{tabular}

Figure 1. Review of sensors [3]

The proposed system uses wired human sensors to detect the heart rate through a wearable device and send the data directly to the cloud. The IoT device enabled by sensors and internet will help to analyze the system on the cloud, the analysis will be performed by keeping the different age group for different heart rate since the heart rate varies either to high level or low level with respect to age as shown in Table 1.

Table 1. Heartbeat according to age range [4]

\begin{tabular}{ccccc}
\hline Age & $20-35$ & $35-50$ & $50-65$ & $65-75$ \\
\hline $\begin{array}{c}\text { Target Heart rate zone at 50 to 85 percent } \\
\text { exertion }(\mathrm{bpm})\end{array}$ & 93 to 170 & 85 to 157 & 80 to 145 & 75 to 132 \\
$\begin{array}{c}\text { Average Maximum heart rate at } 100 \\
\text { percent exertion(bpm) }\end{array}$ & 185 to 200 & 170 to 185 & 155 to 170 & 150 to 155 \\
\hline
\end{tabular}

The heart rate varies according to age, physical activity, air temperature, stress, etc.; the system uses the calibrations to determine the cause of change. The average heart rate goes 150 to $200 \mathrm{bpm}$ as per the age group. The system analyses the pulse and predicts whether the person is getting a heart attack due to a fall in amplitude of heartbeats. If there is a sudden fall in amplitude along with heart rate, then it is a case of a heart attack. The application receives analyzed data and displays the fluctuations. In case, the application senses an emergency situation; it triggers an alert message and email.

This system is a holistic solution intended not only for heart monitoring and alerting in case of an emergency situation, but also includes traffic monitoring system to help navigate the Medical help through the fastest route for providing primary care. The system was designed keeping in view the traffic situation and death ratio due to unavailability of primary treatment which could be relayed to the patient in ambulance itself. Additionally, the system connects and alerts the traffic department to help clear the route, like in case of green corridors, for the ambulance to reach the nearest hospital in the ideal window of 180 minutes as recommended by WHO.

\section{THE PROPOSED SYSTEM VS. TRADITIONAL APPROACH}

Traditionally, patient or caretaker has to inform the hospital or ambulance for the treatment to begin. In India, according to 2013 civil registration data, $27 \%$ of total deaths happen with no medical attention. Therefore, the proposed system tries to prevent cases of heart attacks or cardiac arrest by providing a holistic solution. The system includes a wrist-wearable sensor which captures data along with heartbeat in real time. The data is further analyzed on the cloud using algorithms. In the event of a heart attack, the system triggers alert to nearby Hospital, Ambulance, and the emergency contacts of the patient. Additionally, the system aids the ambulance similar to Ola app, guiding it through the fastest route. 


\section{RESEARCH METHOD}

\subsection{Block diagram}

The Figure 2 illustrates the sensor-enabled Arduino Kit in a watch; wherein Bluetooth transfers the collected data to the Blynk App. The app, in turn, transmits the data to Firebase, a cloud service, for computation. Based on data, Firebase triggers alert to Hospital, ambulance, and emergency contacts. Additionally, the system also enables to manually trigger the alert to emergency contacts in case of any unforeseen emergency.

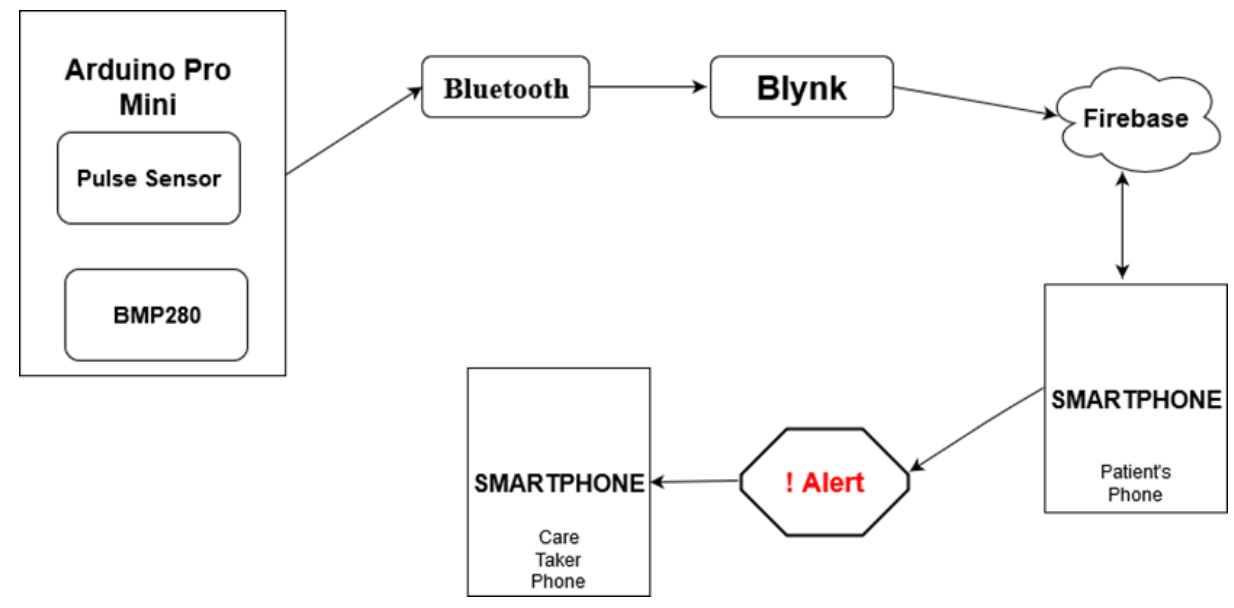

Figure 2. Block diagram of proposed model

\subsection{About System}

The Sensors captures Beats per minute (BPM), air temperature and barometric pressure. In addition to the above data, the system also considers age, maximum and minimum heart rate while analyzing the data in the cloud. Based on the evaluation, an alert is triggered. The system is simple and user-friendly to save the life from a cardiac attack/heart attack.

\subsection{Modules}

a. Sensing Module: is the main module, capturing the data from different sensors connected to Arduino pro mini. The module includes a pulse sensor and temperature sensor.

b. Alert Module: The module will send the alert messages to native, ambulance and hospital by which the patient can get the treatment in the limited period of 180 minutes. This alerting system will not only send the message but also send the mail, and the ambulance will get the complete detail of the patient's location.

\subsection{Frame Work for India Traffic}

This framework will help to assist the shortest route to the ambulance. The concept of the framework is same as that of OLA or Uber except for the choice of driver to accept the request. The Figure 3 illustrates the ambulance controller will send the detail of patient's location to nearest ambulance located in patient's locality by checking all the nearest ambulance in the area. Once the nearest ambulance receives the patient's coordinates, the ambulance controller will start the navigation system showing the fastest route. The controller will have all the information about the ambulance, i.e., the time, distance and current location from the patient house. Ambulance controller then transfers all the details of the ambulance to the traffic controller, which helps the system to monitor the ambulance and clear the ambulance route. With the help of the information sent to the traffic department, arrangements could be made to ensure that the ambulance reaches the hospital in the minimum possible time. Benefits of the framework:

- The patient can reach the hospital in minimum time.

- The life can be saved, since the treatment in cardiac arrest should be given as soon as possible, the primary aid can be provided in the ambulance itself.

- Reduce in death ratio

- The decrease in traffic problem that is faced by ambulance daily due to increasing traffic. 


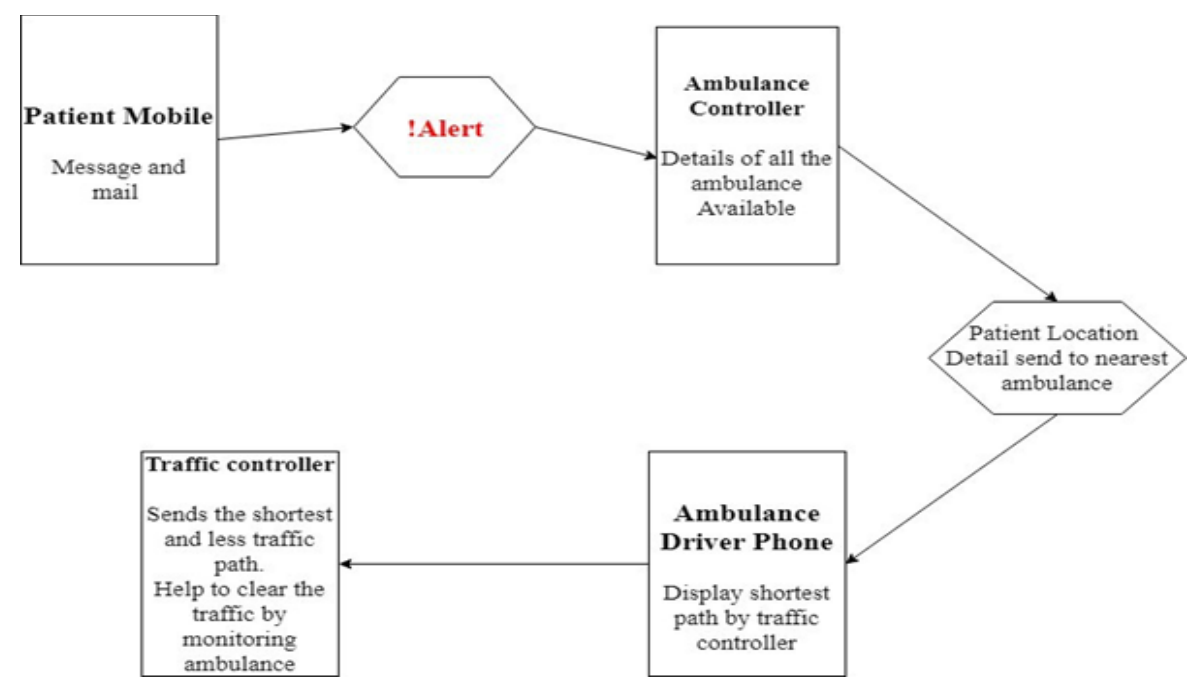

Figure 3. Framework Model

\subsection{Algorithm}

Arduino Ide is installed with window based operating system. The code is written in Arduino IDE. The function of algorithm is to detect the heart rate along air temperature and barometric pressure which tells that whether there is sudden fall of human body and alert the native people via SMS and email alerts in case of emergency. The algorithm is as follow:

Step 1: Start.

Step 2: The heart rate sensor detects the heart rate from the wrist of the user and similarly BMP280 detect the barometric pressure.

Step 3: The output of heart beat sensor is given to Arduino Pro.

Step 4: The algorithm of heart rate attack will be compiled with the output of heart beat sensor and display the number of pulses as output. The output of sensors is stored in the cloud.

Step 5: with the HC-05 Bluetooth, the data will be send to android mobile application where pulse rate and altitude is displayed.

Step 6: If received data from sensors goes below or high from the range which is already stored, then the alert is send to native, ambulance and hospital from android application by itself.

Source code of Bmp280:

void $\operatorname{loop}()$ \{

Serial.print("Temperature =");

Serial.print(bme.readTemperature());

Serial.println("*C");

Serial.print("Pressure = ");

Serial.print(bme.readPressure());

Serial.println(" $\mathrm{Pa}$ ");

Serial.print("Approx altitude $=$ ");

Serial.print(bme.readAltitude(1013.25)); // this should be adjusted to your local forcase Serial.println(“ m");

Serial.println();

delay(2000); // delay of $2 \mathrm{sec}$ \}

This play major role because the value fetched from sensor are checked whether a patient got the heart attack or not by checking the fall in altitude and pressure.

\section{RESULTS AND ANALYSIS}

The Figure 4 shows the connection of all the components to the breadboard. The pulse sensor, BMP280, HC-05 and Arduino pro mini is connected to the breadboard, once the LED is ON the connection is successfully established. The data is sent to the blynk application by HC-05 which is a Bluetooth component to transfer the data from the Arduino kit to mobile or over the internet. 
The data displayed in the blynk application in Figure 5 shows the Altitude which helps to check the sudden fall and the heart rate shows in the bpm (beat per minute). If the Heart rate is normal, i.e. within the specified range than it shows the current health status of the patient. The graph shows the heart rate in the $\mathrm{y}$-axis and the time is on the $\mathrm{x}$-axis.

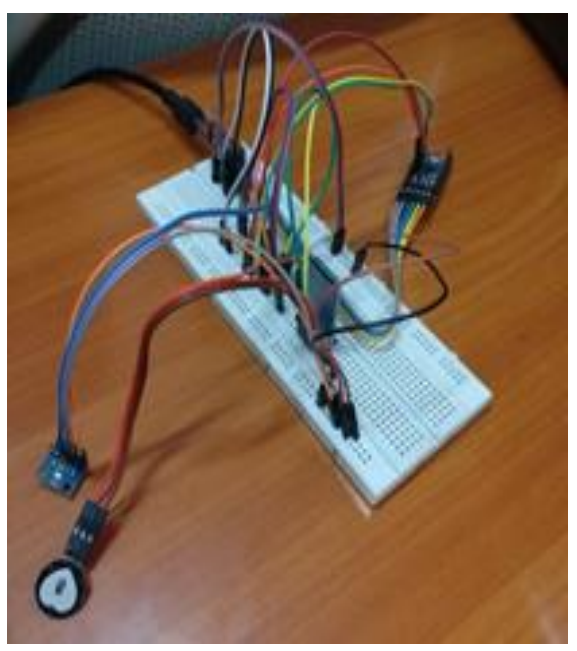

Figure 4. Arduino kit attached with breadboard

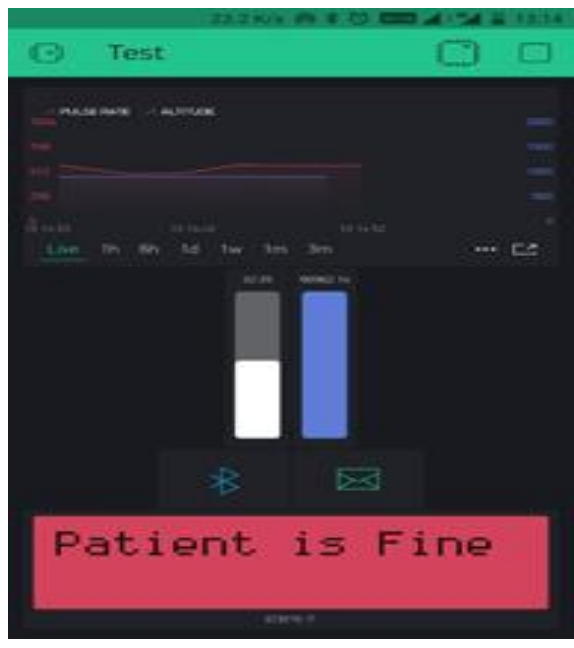

Figure 5. Health Status of the patient

The user can easily see the heart rate of the particular time of a day. Also, the graph will be used to analyze the health status, i.e. at what time the heart rate goes down or high mostly. The analysis can be further used to predict the stress level or exercise time. When the heart rate reaches the critical level as shown in Figure 6, the application triggers messages and email alerts to the registered contacts, nearest hospital, and the ambulance controller. The alert module sends the current location of the patient through which the patient can be reached at the exact location in minimum time.

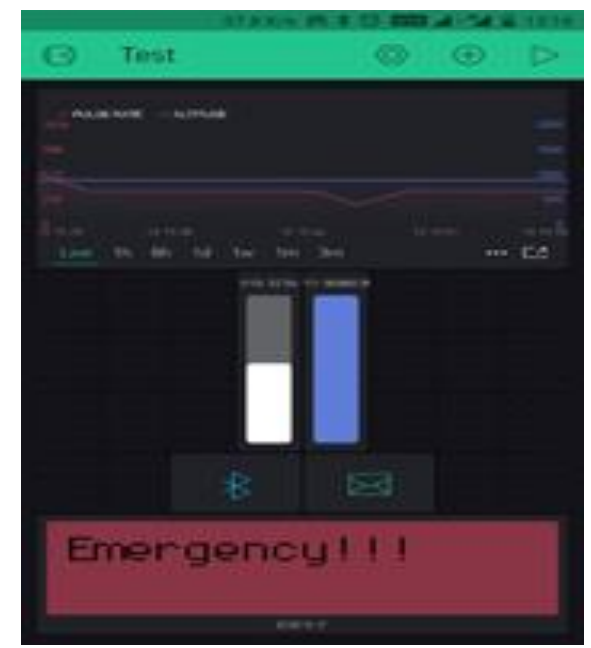

Figure 6. Emergency alarm generated on blynk app

\subsection{Citation}

Siddharth Archarya et al, this paper discussed the health issues of 2015 in India, the different kind of diseases are increasing, and it needs to spread the awareness. The government and health professionals are taking various steps to spread the awareness and implement the health plans to control the health issues. There is a need in India to control the health-related issues since it's a growing country with a daily increase in numbers of population count [5]. 
S. M. Riazul Islam et al, covers the IoT healthcare network which tells the issues and description of each layer. The paper tells about IoT healthcare services, and application that can monitor the health of a person and the applications can get the data from different sensors which are monitoring different health issues like glucose level, ECG, blood pressure, body temperature, etc. The different policies and technologies have taken by different countries for working on IoT and health strategies. There are multiple issues and challenges in IoT Healthcare like standardization, platforms, new disease and disorders, continuous monitoring, scalability, data protection, etc [6].

Shinde Sayali P and Phalle Vaibhavi N., presented the views on IoT which is vast terminology which is helpful in the various field among that it is needed to incorporate new technologies in Healthcare system. The survey paper shows the current need for up-gradation in the existing system of healthcare systems. The survey done on the whole medical system shows un-prosperous maintenance and unutilized technology. The awareness is much needed to spread but with awareness; it is needed that the existing system is upgraded and new technologies should be integrated to monitor and perform the predictions [7].

Asha S N and Brundha Elci J, presented the idea of the system that consist of 4 sensors to monitor the health in a way where the heart rate, body temperature, blood pressure and blood glucose is checked and the data is recorded in the android application which can be monitor by healthcare professionals to diagnose and advice the patients about their health issues. The smart sensor system is capable of detecting the medical distress by using a blood-oxygen sensor which measures the amount of oxygen in the blood [8].

Gowrishankar S. et al, developed a system which takes the pulse rate from the fingertip of the person and the body temperature of the person. The model is consisting of 2 sensors, i.e., a pulse rate sensor, and LM35; the heartbeat is measured in bpm which is further used to make the analysis. The data is transferred over the internet to store it in the server so the further analysis can be performed by a physician or specialist. Their future work is to integrate more sensors so the health of the person can be performed and the real data analysis for better precautions and services [9].

Aboobacker Sidheeque et al, implemented the IoT based heart rate monitoring system where the user is initially setting the low and high heart rate; the system will monitor the heart rate on the based values fed to the system. After the limits are stored in database, the system will send alerts to the controller over the internet to doctors and concerned users. The user can see the live heart rate on the Android application integrated into the system [10].

Chao $\mathrm{Li}$ et al, proposed the system that consist of three layers which are helping to sense the data and transfer the data. The system developed by them will monitor patient blood pressure, ECG, SpO2 which will help to monitor the health of the patient. The data transmission is provided in four different ways which are real-time continuous data, data transmission in special periods, event triggered and on patient demand. Their plan is to integrate the data stream management system to handle the data since the data generated by the system will be more and continuous [11].

Mamidi Manisha et al, it is an IoT system which not only monitors the heart rate but also performs the heart disease analysis using the data collected so as to save patient's life. They mainly focus on the old age people who usually get heart disease the system measures the heart rate and blood pressure which is helpful to send the alert messages to mobile phones of concerned user's. The big data analysis is performed by using Hadoop algorithms to give the flexibility and transparency to the patients [12].

Sharanbasappa Sali and Parvathi C. S, implemented the system which tells how the ZigBee transmitter can be helpful to send the data, the sensors i.e. heart rate sensor and temperature sensor will be attached to the Arduino board and the data is sent through GSM module. The patient can set the parameters values according to the previous health status, and the data will be analyzed by applying $\mathrm{K}$ mean algorithm in Mat-Lab tool, and if the current values of parameters go above the threshold value, then the alert message is sent [13].

Ravi Kumar Sah et al, implemented the system which is useful for Heart Failure patients since it monitors heartbeat in real time. The system is connected to the ECG sensor which is attached to the patient body and records the heartbeat values; the Bluetooth sensor HC-05 is to establish the communication and transferring the data over the short distance devices. The sensors will be connected to microcontroller which takes the readings and with the help of Bluetooth the data is sent to mobile application where analysis is performed, and if the current values go above or below the threshold value then it will send the alert to the life of patient can be saved and the treatment can be provided on time. The system helps the doctor to provide the treatment and precautions by analyzing the values collected by the sensors. Since the system is connected to the mobile application so at the time of help the current location of the patient is sent to the concerned users [14]. 


\section{CONCLUSION}

The proposed system is though not yet implemented however preliminary results shows that life of a patient can be saved if the treatment is provided on time. The system is tested with a limited number of people; the system is taking correct values from the patient body and triggering the alert if any emergency is sensed. The framework aims at reducing the deaths caused by cardiac arrest especially when the patient is in sleep or unconscious. Moreover, the proposed framework will help to improve the faster accessibility of ambulance in the city. The proposed system is user-friendly and economical so that the CVD patients can afford and could keep close tabs; so, in case of emergency help could reach them through the fastest route.

\section{REFERENCES}

[1] http://www.indiaspend.com/indias-great-challenge-health-sanitation/heart-disease-kills-1-7-million-indianscontinues-to-be-top-killer-in-2016.

[2] https://www.hindustantimes.com/india-news/more-than-50-of-heart-attack-cases-reach-hospital-late-govt-datashows/story-penFdsewgGwpIwiQnRDoLJ.html.

[3] Appelboom, et al., "Smart wearable body sensors for patient self-assessment and monitoring," Archives of Public Health, vol. 72, pp. 28, 2014.

[4] https://www.medicalnewstoday.com/articles/235710.php.

[5] S. Acharya, et al., "Current status of major health problems of India," International Journal of Current Research, vol/issue: 7(10), pp. 21873-21877.

[6] Hossain, et al., "The Internet of Things for Health Care: A Comprehensive Survey," IEEE Access, vol. 3, pp.678-708.

[7] S. Sayali P. and P. Vaibhavi N., "A Survey Paper on Internet of Things based Healthcare System,” vol/issue: 4(4), Jan 2017.

[8] Asha S. N., "Human Activity Monitoring Using the Wearable Sensors in Health care," International Journal of Science, Engineering and Technology Research (IJSETR), vol/issue: 5(2), Feb 2016.

[9] Gowrishankar S., et al., "IoT based Heart Attack Detection, Heart Rate and Temperature Monitor," Jul 2017.

[10] A. Sidheeque, et al., "Heartbeat Sensing and Heart Attack Detection using Internet of Things: IoT," Apr 2017.

[11] C. Lia, et al., "The IoT-based heart disease monitoring system for pervasive healthcare service," Dec 2017.

[12] M. Manisha, et al., "IOT on Heart Attack detection and Heart Rate Monitoring," International Journal for Innovations in Engineering and Technology, Aug 2016.

[13] S. Sali, et al., "Integrated Wireless Instrument for Heart rate and Body Temperature Measurement," 2017 2nd International Conference for Convergence in Technology (I2CT), 2017.

[14] R. K. Sah, et al., "Heart Disease Monitoring System Using Contact Measure of Stability index," 2017 2nd IEEE International Conference On Recent Trends in Electronics Information and Communication Technology (RTEICT), India, May 2017.

\section{BIOGRAPHIES OF AUTHORS}
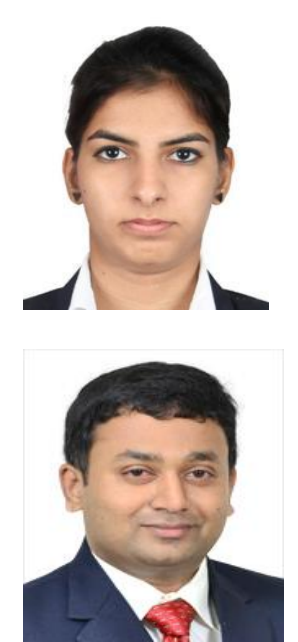

Khushboo Bhagchandani completed Master of Computer Science from CHRIST (Deemed to be University). She obtained Bachelor Degree in Computer Application from Global College (India). As a part of studies she has done research to improve health sector with the help of technologies. She is being guided by a research scholar Dr D. Peter Augustine.

Dr D. Peter Augustine is a research scholar and associate professor in CHRIST (Deemed to be University). His domain is cloud computing and health care. He has published research articles in various journals in his domain. 